\title{
Los diuréticos en bajas dosis son la mejor primera línea de tratamiento antihipertensivo
}

Health outcomes associated with various antihypertensive therapies used as first-line agents: a network meta-analysis. Psaty BM, Lumley T, Furberg CD, Schellenbaum G, Pahor M, Alderman MH, Weiss NS. JAMA. 2003 May 21;289(19):2534-44.

\section{Objetivo}

Resumir la evidencia referente la seguridad y eficacia de los antihipertensivos de primera línea de mayor uso:diuréticos tiazídicos a bajas-dosis (12,5-25mg/día) vs otros cinco tratamientos:Beta-Bloqueantes (BB), Inhibidores de la Enzima Convertidora de Angiotensina (IECA), Bloquenates Cálcicos (BC), Alfa-Bloqueantes y Antagonistas de los receptores de la Angiotensina-II (ARA-II).

Fuente y selección de estudios

Meta-análisis anteriores, MEDLINE y revisiones de 1995 a 2002. Se identificaron ensayos clínicos controlados y aleatorizados (ECCA) a largo plazo, de más de 400/personas-año (controles: placebo/notratamiento $u$ otros tratamientos activos) que evaluaran eventos cardiovasculares mayores (ECVM) como resultado.

Se incluyeron estudios en inglés y en otros idiomas.Se los excluyó si evaluaban pacientes con infarto de miocardio (IM) o insuficiencia cardíaca, en cese tabáquico o con hipolipemiantes, excepto que hubieran tenido un diseño factorial* .

\section{Extracción de datos}

Dos investigadores independientes extrajeron los datos de este Network Meta-análisis* (Meta-análisis en "Red" traducido al castellano) de antihipertensivos de primera línea que combinaran comparaciones directas (ej.una droga contra otra) más comparaciones indirectas (aprovechando toda la información de los tratamientos en común). Los resultados evaluados fueron enfermedad coronaria (EC): IAM fatal, no fatal y muerte coronaria; insuficiencia cardíaca (IC):IC fatal y no fatal; accidente cerebrovascular (ACV): ACV fatal y no fatal; ECVM:los tres anteriores más mortalidad por otras enfermedades cardiovasculares (CV); mortalidad CV y mortalidad total.

\section{Resultados}

Se combinaron datos de 42 ECCA (192 478 pacientes seguidos entre tres y cuatro años).En todos resultados evaluados los diuréticos fueron superiores al placebo. $\mathrm{Vr}$ cuadro 1.

Cuadro 1:resultados de las comparaciones entre diuréticos y placebo.

\begin{tabular}{l|l|c}
\hline Diuréticos vs Placebo & RR (IC95\%) & $\mathbf{p}$ \\
\hline Enfermedad coronaria & $0,79(0,69 \mathrm{a} 0,92)$ & 0,002 \\
\hline Insuficiencia cardíaca & $0,51(0,42 \mathrm{a} 0,62)$ & $<0,001$ \\
\hline Accidente cerebrovascular & $0,71(0,63 \mathrm{a} 0,81)$ & $<0,001$ \\
\hline Evento cardiovascular mayor & $0,76(0,69 \mathrm{a} 0,83)$ & $<0,001$ \\
\hline Mortalidad cardiovascular & $0,81(0,73 \mathrm{a} 0,92)$ & 0,001 \\
\hline Mortalidad total & $0,90(0,84 \mathrm{a} 0,96)$ & 0,002 \\
\hline
\end{tabular}

Ninguno de los antihipertensivos de primera línea fue estadísticamente superior a los diuréticos a bajas-dosis en resultado alguno. Comparados con los $\mathrm{BC}$, los diuréticos presentaron menor RR de ECVM: $0,94(0,89$ a 1,00$)$ y de IC $0,74(0,67$ a 0,81$)$; comparados con los IECA, menor RR de IC 0,88 $(0,80$ a 0,96), ECVM 0,94 $(0,89$ a 1,$00 ; p=0,04)$ y ACV $0,86(0,77-0,97)$; comparados con los $B B$, menor RR de ECVM 0,89 $(0,80$ a 0,98$)$ y con los Alfa-bloqueantes menor RR de ECVM 0,84 (0,75 a 0,93). No hubo diferencias significativas entre Dihidropiridinas y No-dihidropiridina por lo que los BC se analizaron juntos. Los cambios de Tensión Arterial (TA) fueron similares entre grupos con una tendencia no significativa de menor TA con diuréticos que con otros antihipertensivos.

\section{Conclusión}

Los diuréticos a bajas-dosis son los antihipertensivos de primera línea más eficaces. Previenen la morbimortalidad CV. Las guías de práctica deberían reflejar esta evidencia y los futuros ECCA deberían usar diuréticos a bajas-dosis como comparación estándar.

Fuente de financiamiento: National Heart, Lung, and Blood Institute, National Institute on Aging y American Heart Association (AHA).

\section{Comentario}

Dado el complejo arsenal de estudios que comparan tratamientos antihipertensivos, es difícil establecer la relación entre los daños y los beneficios de algunos agentes específicos. Las comparaciones entre dos tratamientos (ambos efectivos frente al placebo) requieren gran " $n$ " y seguimiento prolongado para demostrar diferencias en resultados clínicos relevantes, y hasta los meta-análisis clásicos tienen poder insuficiente debido a la dispersión en múltiples comparaciones. Por esta razón se utilizó el "meta-análisis en red" para evaluar la totalidad de la evidencia disponible sobre antihipertensivos de primera línea en términos de enfermedad CV mayor y mortalidad.

El JNC6 ${ }^{1}$ había establecido en 1997 que no había ventajas con los nuevos agentes pues las tiazidas y los BB habían probado disminuir la morbimortalidad frente al placebo. Si bien el $\mathrm{JNC}^{2}$ recomienda a los diuréticos tiazídicos como drogas de primera elección en pacientes sin comorbilidades (sólos o asociados a otras drogas) esta recomendación se basó principalmente en la cantidad de evidencia acumulada y los menores costos los diuréticos. Sin embargo hasta el presente estudio no había evidencia clara de superioridad de los mismos frente a otros tratamientos. Si bien el ALLHAT (mayor estudio comparativo realizado) fue consistente con estos resultados ${ }^{3}$ no debe olvidarse que la cuestión no estaba resuelta ya que, por ejemplo, el estudio ANBP2 mostraba conclusiones divergentes de beneficios de los IECA frente a diuréticos ${ }^{4}$. Antes de combinar toda la evidencia mediante el "meta-análisis en red" se analizaron por separa- do los resultados del ALLHAT y se hicieron comparaciones directas e indirectas entre tratamientos con resultados similares (baja incoherencia*) en sentido y magnitud, lo que avala la estrategia de analizarlos conjuntamente y, por ende, la conclusión acerca de la superioridad de los diuréticos en el tratamiento inicial de la hipertensión arterial.La tendencia a menor TA alcanzada con los diuréticos no podría explicar las diferencias halladas.Por ejemplo, datos del Framingham Heart Study ${ }^{5}$ muestran que si se ajustara por el descenso de $2,4 \mathrm{mmHg}$ de la TA alcanzada con los diuréticos respecto de los BC, solo se explicaría parte de el efecto protector sobre la IC (el RR IC cambiaría de 0,74 a 0,77).

En pacientes con ciertas comorbilidades como diabetes sería razonable iniciar con IECA, especialmente si son añosos;y en el caso de IC o historia de IM, tanto los IECA como los BB ofrecerían ventajas frente a los diuréticos. De todas formas, independientemente de la terapéutica inicial, no debemos perder de vista que frecuentemente se necesitan asociaciones que, por todo lo expuesto, no debieran obviar los diuréticos y por supuesto alcanzar las metas individuales de control de la HTA.

Conclusiones del comentador: todos los antihiper tensivos son altamente efectivos frente al placebo. Este meta-análisis aporta evidencias claras de superioridad de los diuréticos como tratamiento de primera elección para la HTA, especialmente en pacientes sin comorbilidades.

Dr. Agustín Ciapponi [ Unidad de Medicina Familiar y Preventiva. Hospital Italiano de Buenos Aires ]

*Ver Glosario

\section{Referencias}

1- The sixth report of the Joint National Committee on prevention detection, evaluation and treatment of high blood pressure.Arch Intern Med 1997; 157:2413-46

2- Aram V.Chobanian, George L. Bakris, et al.The Seventh Report of the Joint National Committee on Prevention, Detection, Evaluation, and Treatment of High Blood Pressure:The JNC 7 Report. JAMA.2003;289:2560-2571.

3- Ciapponi A. Comentario de Similar eficacia de las tiazidas y otras drogas como tratamiento de primera línea de la Hipertensión Arterial Evidencia en atención primaria 2003 Marzo - Abril Vol 6 Nro 2:38. Comentado de:Major Outcomes in high risk hypertensive patients randomized to Angiotensin-Conv e rting Enzyme Inhibitor or Calcium Channel Blocker vs Diuretic:the Antihypertensive and Lipid Lowering Heart Attack Trial (ALLHAT) , JAMA 2002;288:2981-2997

4- Ciapponi A. Comentario de Los inhibidores de la enzima de conversión fueron algo superiores a las tiazidas Evidencia en atención primaria 2003 Marzo - Abril Vol 6 Nro 2:39. Comentado de:Wing L, Reid C, Ryan P, et al.A comparison of Outcomes with Angiotensin Conv e rting Enzyme Inhibitors and Diuretics for Hypertension in the Elderly.N Engl J Med 2003;348:583-9255- Cupples LA, D'Agostino RB.Some risk factors related to the annual incidence of cardiovascular disease and death using pooled repeated biennial measurements:Framingham Heart Study, 30-year follow-up. In: KannelWB, Wolf PA, Garrison RJ, eds. The Framingham Study:an Epidemiological Investigation of Cardiovascular Disease.Bethesda, Md:National Institutes of Health; 1987. NIH No. $87-2703$. 